\title{
Measurements of plasma parameters in the divertor island of Wendelstein 7-X through line-ratio spectroscopy on helium
}

\author{
T. Barbui ${ }^{1,2}$, M. Krychowiak ${ }^{3}$, O. Schmitz ${ }^{1}$, S.A. Bozhenkov ${ }^{3}$, E. Flom ${ }^{1}$, G. Fuchert ${ }^{3}$, C. Killer ${ }^{3}$, R. König ${ }^{3}$, \\ M. Jakubowski ${ }^{3}$, J. M. Muñoz Burgos ${ }^{4}$, E. Pasch ${ }^{3}$, E.R. Scott ${ }^{3}$ and the W7-X Team ${ }^{3}$ \\ ${ }^{1}$ Department of Engineering Physics, University of Wisconsin-Madison, Madison, WI 53706, USA \\ ${ }_{2}^{2}$ Princeton Plasma Physics Laboratory, Princeton, NJ 08540, USA \\ ${ }^{3}$ Max-Planck-Institut für Plasmaphysik, 17491 Greifswald, Germany \\ ${ }^{4}$ Astro Fusion Spectre LLC, San Diego, CA 92127, USA \\ E-mail: tbarbui@pppl.gov
}

\begin{abstract}
Electron temperature $T_{\mathrm{e}}$ and density $n_{\mathrm{e}}$ have been measured in the divertor island of Wendelstein 7-X by means of a new thermal He-beam system utilizing line-ratio spectroscopy. This system is equipped with a poloidal arrangement of five gas valves that allows to infer 2D plasma profiles $T_{\mathrm{e}}(R, Z)$ and $n_{\mathrm{e}}(R, Z)$ across the magnetic island forming the island divertor. A local maximum in $T_{\mathrm{e}}(R, Z)$ was measured along the flux surfaces located between the island center and the outer separatrix. In the island center, characterized by closed field lines, a clear local minimum in $T_{\mathrm{e}}$ was measured, indicating that no direct heat deposition occurs in this region and that the perpendicular heat flux from the core plasma into this domain is small. Increasing the island size leads to a reduction of the connection length in the scrape-off layer and to a shift of the island center towards the divertor target. In this scenario, the $T_{\mathrm{e}}(R, Z)$ are reduced in the entire island domain. Density profiles are found flat across the divertor island for both island size scenarios. These findings are supported by similar observations made with a reciprocating probe plunging through the same scrape-off layer island at the mid-plane of W7-X representing the upstream position in this $3 \mathrm{D}$ divertor geometry.
\end{abstract}

\section{Introduction}

One of the most significant challenges that need to be addressed in the quest for fusion energy is heat and particle exhaust. In the magnetic confinement devices, both tokamaks and stellarators, divertors provides a well defined interaction zone between the exhaust plasma and the material of the divertor targets. For stellarators, the concept of the island divertor is being explored at the Wendelstein 7-X (W7-X) stellarator for the first time in this scale and towards quasi steady state conditions [1] [2]. Magnetic islands are utilized as the plasma core to material interface. These islands are formed on a low order rational magnetic surface in the plasma edge and hence, they are intrinsically present in the magnetic field configuration of W7-X. To exhaust the heat and particles coming from the confined region, these islands are intersected by target plates which forms the diverting action on the heat and particle fluxes. The open field lines inside the island guide the heat entering the island across the inner separatrix towards the divertor targets. The islands form the scrape-off layer (SOL) between the core and the plasma-surface interaction region, preventing the core plasma from a direct exposure to the recycling neutrals and sputtered impurities [3].

The stellarator Wendelstein 7-AS was the first to explore the concept of the island divertor [4]. Wendelstein 7-X is its successor. It is a large optimized superconducting stellarator of the HELIAS type with a strongly varying plasma cross-section and low shear ( $R=5.5 \mathrm{~m}, a=0.55 \mathrm{~m}, B_{0}=3 \mathrm{~T}$, five periods) [5]. Since 2017 the device has been equipped with uncooled graphite divertor units which implement the island divertor concept [6]. In the standard $m / n=5 / 5(1=1)$ magnetic configuration five islands are present in the boundary domain. They form the 
SOL region, defining five toroidally closed helical magnetic flux tubes. The island inner separatrices define the last closed flux surface (LCFS) while the outer separatrices are intersected by target plates and define the position of the heat and particle exhaust deposition. In this way the plasma flow is concentrated on the target plates and the plasma-wall interaction is separated from the plasma core [7]. Additional divertor control coils are available to control the variation of the connection length $\left(L_{c}\right)$ and modify the distance between target plates and separatrix by changing the island size [8]. When a positive current is driven in the divertor control coils, a radial magnetic perturbation $B_{\mathrm{r}}$ is induced in the plasma which increases the island width and reduces the connection lengths inside the island.

The heat and particle transport across the island is governed by the balance of parallel and perpendicular transport. Contrary to the single-null divertor of tokamaks, in which short $L_{\mathrm{c}}(O(10 \mathrm{~m}))$ prevail and where the parallel transport dominates, in the island divertor the role of cross-field transport is increased due to the much longer $L_{\mathrm{c}}$ $(O(100 \mathrm{~m}))$. Accordingly, depending on the actual SOL plasma conditions, different magnetic flux tubes have different parallel-to-perpendicular ratios, which can be externally controlled by $B_{\mathrm{r}}[9]$.

For the understanding of the physics of the island divertor, it is of fundamental importance to directly measure $T_{\mathrm{e}}(R, Z)$ and $n_{\mathrm{e}}(R, Z)$ inside the island. The measurement of these parameters, and comparison to $3 \mathrm{D}$ edge transport modeling like for instance EMC3-EIRENE, allows to shed light into the mechanisms that govern the transport of heat and particle inside the island. Moreover, the measurement of $T_{\mathrm{e}}(R, Z)$ and $n_{\mathrm{e}}(R, Z)$ inside the island is fundamental to access the transition between different divertor plasma regimes, such as attached and detached. This work presents the first measurements realized through line-ratio spectroscopy on a thermal He-beam of 2D (radial + poloidal) profiles of $T_{\mathrm{e}}(R, Z)$ and $n_{\mathrm{e}}(R, Z)$ in the island divertor [10]. The He-beam measurements presented in this paper were carried out in the standard magnetic configuration without and with application of the divertor control coils to vary the island size. Additional measurements are presented from other edge diagnostics located at upstream positions and they are compared to the He-beam data.

\section{Experimental results}

\section{Description of the diagnostics}

At W7-X the He-beam diagnostic is installed in one of the five bottom divertors. With this diagnostic $T_{\mathrm{e}}(R, Z)$ and $n_{\mathrm{e}}(R, Z)$ are inferred through the line-ratio spectroscopy technique on neutral helium [11]. A thermal helium beam is injected into the plasma from five different valves equipped with nozzles located at different poloidal positions in the horizontal divertor target (poloidal separation of the valves is $2.5 \mathrm{~cm}$ ). An array of lines-of-sight looking perpendicularly to the beam collects the emission of He I lines. By applying a recently updated hybrid time dependent/independent helium line-ratio solution, it is possible to determine $T_{\mathrm{e}}$ and $n_{\mathrm{e}}$ by the ratio of selected emission lines [12]. This model is based on one that was extensively applied at TEXTOR [11] and has recently also been used at ASDEX-Upgrade [13]. The model used to infer $T_{\mathrm{e}}$ and $n_{\mathrm{e}}$ presented here includes high Rydberg states. A detailed description of the diagnostic including the injection and the observation system and the used atomic model is reported in other publications [14] [10]. $T_{\mathrm{e}}$ and $n_{\mathrm{e}}$ radial profiles are inferred from 0.8 to $9 \mathrm{~cm}$ above the target with a spatial separation between the lines-of-sight $(\mathrm{LoS})$ of $5.4 \mathrm{~mm}$ and a spatial resolution of each LoS of 2-3 mm. In Figure 1(a), the position of the He-beam LoS for the five different valves is shown, superimposed on the Poincare plot for the standard magnetic configuration without and with application of the divertor control coils $\left(I_{\mathrm{cc}}\right)$. The He-beam setup enables to measure the radial profiles at 5 poloidal positions. This yields a map of $T_{\mathrm{e}}(R, Z)$ and $n_{\mathrm{e}}(R, Z)$ across a portion of the island: radially along a $\sim 8 \mathrm{~cm}$ profile and poloidally from the O-point up to $10 \mathrm{~cm}$ towards the baffle side. Error bars shown for the He-beam measurements in this work are experimental uncertainties only. Systematic uncertainties derived from the current atomic model are at 

for $T_{\mathrm{e}}$ and $10 \%$ for $n_{\mathrm{e}}$ [11].

In Figure 1 $(b)$ and $(c)$, the target-to-target connection lengths $\left(L_{c}\right)$ are shown in the divertor domain covered by the He-beam. For the case where no control coils current is applied (Figure 1(b)), connection lengths between 350 and $400 \mathrm{~m}$ are found in the island region for the standard magnetic configuration. The island center is defined by a region of closed field lines (the white color in the colored map indicates infinite connection lengths). A region of very low connection lengths $(<30 \mathrm{~m})$ exists in the far SOL, which represents the shadowed region where the field lines are intercepted by the targets before they complete one revolution around the torus. The connection lengths in the island region drop to 200-250 m (Figure 1(c)) after application of positive current $I_{\mathrm{cc}}=2 \mathrm{kA}$ in the control coils. The island O-point moves radially outwards and the region of closed field lines in the island center shrinks considerably. In this configuration the divertor island width increases by a 3-4 centimeters, its inner separatrix is pushed inwards by $\sim 2 \mathrm{~cm}$, the intersection of its outer separatrix with the horizontal target moves $\sim 6 \mathrm{~cm}$ towards the pumping gap on the left side and the same amount towards the baffle on the right side (see Figure 1(a)).

At the outboard mid-plane, a multi-purpose manipulator is installed [15]. It serves as a carrier for various probe heads equipped with electrical and magnetic probes. It can be plunged through the island chain until the separatrix with up to three strokes per discharge. In the experiments described here, the manipulator was equipped with the electric probe IPP-FLUC1 which contains 28 electric pins for various measurements of SOL profiles and fluctuations [16]. $T_{\mathrm{e}}$ and $n_{\mathrm{e}}$ profiles are measured by a triple probe setup [17]. Electron density is determined by the measure of the ion saturation current, knowing the effective probe surface area and the speed of sound. The latter contains variable that are not yet well characterized for the edge of W7-X, in particular the effective charge state $Z_{\text {eff }}$ and the ion mass $m_{\mathrm{i}}$ which are subject to the impurity content as well as the ion temperature. Here we assume $Z_{\mathrm{eff}}=1, m_{\mathrm{i}}=m_{\mathrm{p}}$ and $T_{\mathrm{i}}=T_{\mathrm{e}}$. With these simple assumptions the plasma density is probably overestimated by 15 to $30 \%$ [18]. The probe plunges across the island about $10 \mathrm{~cm}$ above the O-point, from the far scrape-off layer up to $2-3 \mathrm{~cm}$ before the separatrix. Figure $2(a)$ and $(b)$ show the target-to-target connection lengths in the island region where the probe measures. Again we notice a strong reduction in the connection length and an outward movement of the island when moving from the configuration without control coils $(a)$ to the one with control coils (b).

In the standard $m / n=5 / 5$ magnetic configuration used here, the two diagnostics are connected by the same magnetic flux tube. They intersect the same magnetic island as shown in Figure 3(a) and $(b)$. Here, the projections of the flux tube started at the reciprocating probe position are shown at the He-beam cross-section for the two magnetic island divertor configurations without and with application of control coils.

In order to further benchmark the He-beam measurements, two edge channels of the Thomson scattering system [19] from the low field side are also considered. These channels are located in between two edge islands. Projections of magnetic flux tubes originating from these channels are shown as yellow dots in Figure 3(a) and (b). 
Figure 1. (a) Poincare plot at the position of the He-beam in the bottom divertor module for the standard magnetic configuration without (blue) and with (red) application of the control coils. The five nozzles are drawn as magenta segments. Collection optics and optical fibers are located in a port between the vertical and the horizontal target (port AEI30). The points where the LoS intercept the beam are shown as green dots. Horizontal, vertical and baffle targets are plotted in black. LCFS for both configurations is drawn as a thick line. $(b)-(c)$ Target-to-target connection lengths in the area enclosed in the black box in $(a)$ for the configuration without $(b)$ and with $(c)$ application of control coils. White color indicates closed field lines. Poincare plots and connection lengths shown in these and in the next figures are computed using ideal coil geometry, main coils currents as in the experiment EJM +252 with error field correction and control coils current $I_{\mathrm{cc}}=0$ and $2 \mathrm{kA}$.. Toroidal plasma current is set to $3 \mathrm{kA}$. 

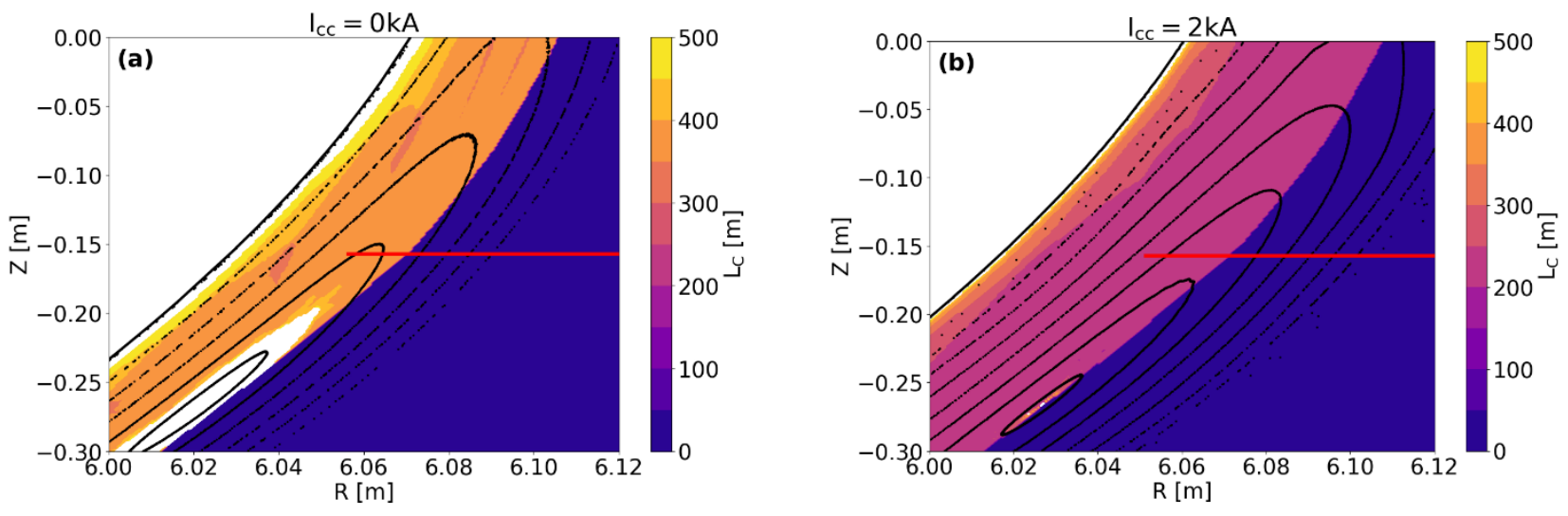

Figure 2. Target-to-target connection lengths in the mid-plane island region crossed by the probe for the configuration without $(a)$ and with $(b)$ application of control coil current. The probe path is indicated by the red line. White color indicates closed field lines. Poincare plots for the two configurations are shown as black dots.
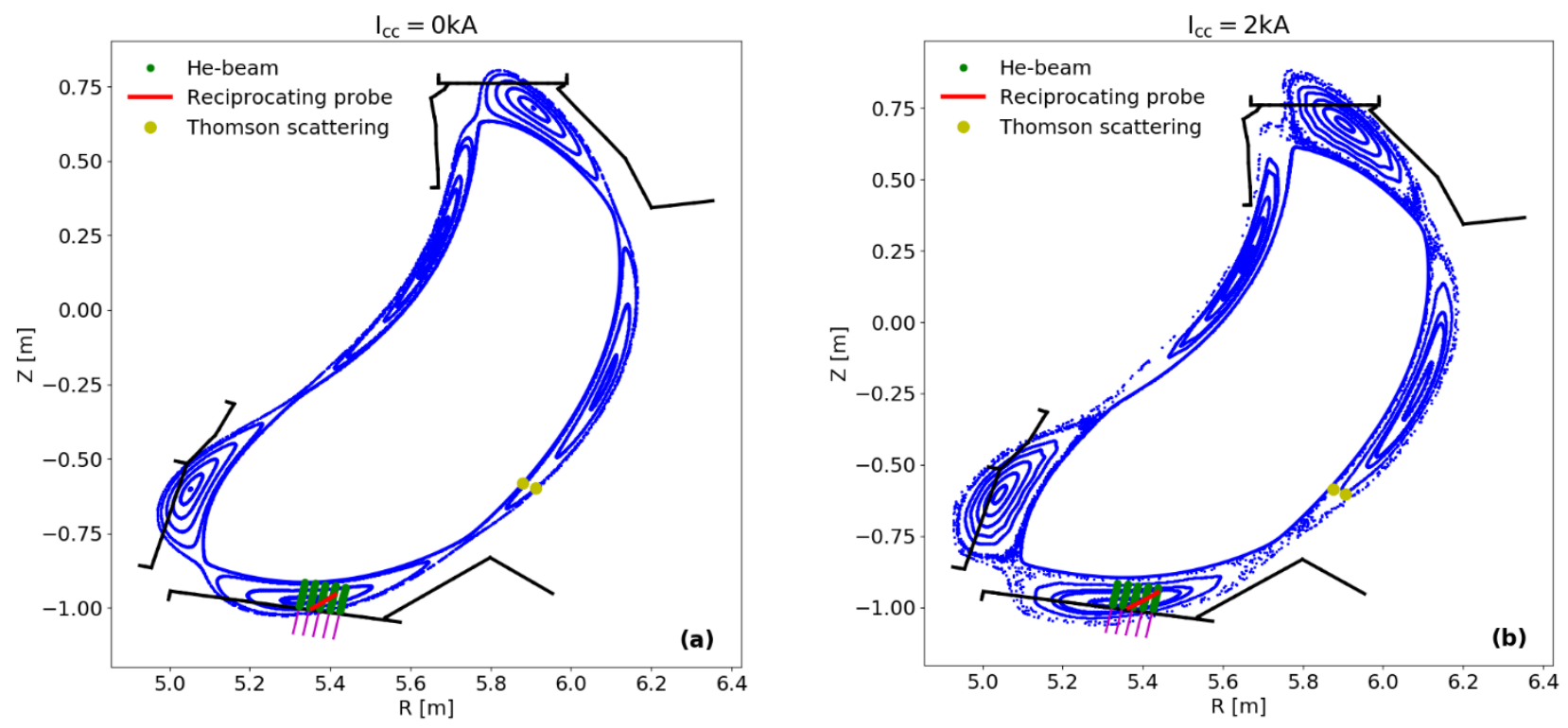

Figure 3. Poincare plots at the poloidal cross-section of the He-beam for the standard magnetic configuration without ( $a$ ) and with $(b)$ application of the control coils. The five nozzles are drawn as magenta segments and the points where the LoS intercept the beam are shown as green dots. Projections of flux tubes originating from the plunging path of the probe are shown as a red line. Projections of flux tubes originating from the edge channels of the Thomson scattering system (low field side) are shown as yellow dots. Flux tube tracing shown here is computed using ideal coil geometry, main coils currents as in the experiment EJM+252 with error field correction and control coils current $I_{\mathrm{cc}}=0$ and $2 \mathrm{kA}$. Toroidal plasma current is set to $3 \mathrm{kA}$.

\section{Description of the experiments}

The experiments described in this work are conducted in hydrogen with ECRH power of $4.5 \mathrm{MW}$ at a line average electron density of $3 \times 10^{19} \mathrm{~m}^{-3}$. Divertor control coils are used in some of these experiments with a positive current of $2 \mathrm{kA}$. Reference time traces of the main plasma parameters for one discharge are plotted in Figure 4. The He- 
beam is switched on after $2 \mathrm{~s}$ from the beginning of the discharge with the first injection being launched from the first valve on the right side. Each valve is active in a $0.5 \mathrm{~s}$ time interval, during this period the valve is operated in a modulated way with a 50/50 duty cycle (75 ms on-time, $75 \mathrm{~ms}$ off-time). During off-time no gas is being injected and this phase is used to subtract the background radiation. During on-time $\mathrm{He}$ is injected and the He intensity is integrated for $25 \mathrm{~ms}$. Due to the non-synchronization between valve and camera timing, and a relatively slow opening time of the valves ( $12 \mathrm{~ms}$ ), only two He intensity measurements are carried out per on-time period of the valve. This provides a total of six He intensity measurements per valve during the $0.5 \mathrm{~s}$ valve cycle, which translates in six $T_{\mathrm{e}}(R, Z)$ and $n_{\mathrm{e}}(R, Z)$ radial profiles per valve, corresponding to 16 data points each. The gas injections are carried out from all 5 valves in sequence, from the right to the left valve. During the time interval of these five helium beam measurements, the reciprocating probe plunges are executed.

The standard magnetic configuration is characterized by the evolution of significant bootstrap currents during the discharge, reaching up to $10 \mathrm{kA}$ for longer discharges (>10 s). The typical toroidal plasma current for the discharges under consideration is shown in Figure 4(e). This current has an impact on the magnetic field structure and consequently on the position and connection length of the edge islands. Field line calculations show that the island is slightly shifted inwards and the connection length is increased in the island at high plasma currents. For high plasma currents above $6 \mathrm{kA}$ these modifications were seen to considerably modify the SOL plasma profiles measured with the mid-plane reciprocating probe and divertor diagnostics [18]. The island shift at the He-beam location has been quantified in $2.5 \mathrm{~mm}$ for each $\mathrm{kA}$ of plasma current. The increase in $L_{\mathrm{c}}$ is about $25 \mathrm{~m}$ per $\mathrm{kA}$ in the SOL. In the experiments considered here the He-beam measurements are carried out in the first part of the discharges $(2<\mathrm{t}<5.6 \mathrm{~s})$ and the value of the toroidal plasma current grows in such interval from 2 to $4-5 \mathrm{kA}$. Hence, the island is shifted inward by $5-7.5 \mathrm{~mm}$ from the time the measurements are taken from the first valve (on the right) to the last valve (on the left), and the connection length is increased by 50-75 $\mathrm{m}$. Connection lengths, Poincare plots and flux tube mapping shown in this work are computed for an intermediate plasma current of 3 kA. It means that the Poincare plots have an uncertainty in the position of the flux surfaces with respect to the measured points of the different valves. Such uncertainty is however comparable with the spatial resolution of the measurements $(2-3 \mathrm{~mm}) . L_{\mathrm{c}}$ calculations have an uncertainty of $\pm 25-50 \mathrm{~m}$ which is within the width of the color scale intervals of the plots.

In these calculations the considered plasma current is described as a current on the magnetic axis, neglecting any spatial distribution. This simplification is justified by Ampère's Law, since here we are only interested in the effects of the current in the confined plasma on the magnetic field in the SOL, outside the confined plasma [18]. 


\section{Temperature and density in the magnetic island that forms the island divertor}

Radial profiles of the electron temperature and density are measured with the He-beam at different poloidal positions at consecutive times during the discharge. These profiles are used to obtain $2 \mathrm{D}$ maps of $T_{\mathrm{e}}(R, Z)$ and $n_{\mathrm{e}}(R, Z)$ in the divertor region. Firstly, the six $T_{\mathrm{e}}(R, Z)$ and $n_{\mathrm{e}}(R, Z)$ radial profiles measured using the same valve are averaged over the $0.5 \mathrm{~s}$ time window, such that we end up with a single $T_{\mathrm{e}}(R, Z)$ and $n_{\mathrm{e}}(R, Z)$ radial profile per valve consisting of 16 data points. Secondly, we create a grid of equidistant points $(100)$ inside the $(R, Z)$ area delimited by the He-beam LoS. Thirdly, we linearly $2 \mathrm{D}$ interpolate the values of $T_{\mathrm{e}}(R, Z)$ and $n_{\mathrm{e}}(R, Z)$ for the five valves (total number of data points $=16 \times 5)$ on the whole grid of points. With this procedure we realize $2 \mathrm{D}$ maps of $T_{\mathrm{e}}(R, Z)$ and $n_{\mathrm{e}}(R, Z)$, as reported in Figure 5 for the case without and with application of control coils. The superimposed Poincare plots are computed for an intermediate value of the plasma current of $3 \mathrm{kA}$ as explained earlier.

The $T_{\mathrm{e}}(R, Z)$ map for the case with $I_{\mathrm{cc}}=0 \mathrm{kA}$ is shown in Figure $5(a)$ as a distribution of $T_{\mathrm{e}}$ in the poloidal ( $R$-axis) and vertical (Z-axis) direction. The actual $T_{\mathrm{e}}$ values are shown as a color map. When moving from the target towards the LCFS the $T_{\mathrm{e}}$ map features a clear local maximum of $\sim 40-50 \mathrm{eV}$ located along the flux surfaces between the island outer separatrix and the island center. Moving further inwards we found a region of low $T_{\mathrm{e}}$ of $\sim 25 \mathrm{eV}$ located in the island center around the O-point. Then, $T_{\mathrm{e}}$ increases overall in form of an exponential trend when approaching the LCFS.

A possible explanation for the decreasing temperature inside the island center characterized by closed flux surfaces is that this region has its own confinement. No direct heat deposition of the ECRH power occurs in this domain and hence the energy source to this domain is perpendicular transport across the LCFS and across the domain of 
finite connection length $L_{\mathrm{c}}$. This finite $L_{\mathrm{c}}$ domain between the closed flux surface region of the island center and the LCFS of the confined domain is a strong energy sink due to the parallel transport towards the divertor plates. However, because $L_{\mathrm{c}}$ is long, the perpendicular transport seems still to be sufficient to facilitate perpendicular heat influx into the domain of good flux surfaces inside the island. Concerning particle and impurity transport, this local domain is sourced by recycling hydrogen flux and impurities from the divertor targets. Therefore, a small perpendicular energy flux would need to sustain the ionization in this domain. This results in significant local energy losses that need to be balanced by the perpendicular energy influx. This will yield reduced local temperatures inside of the good flux surface domain in the islands. Outside this domain the heat coming from the confined plasma flows parallel along the flux surfaces towards the targets. Temperatures measured outside the island center are found higher and the local maxima in the profiles are located along the flux surfaces close to the island outer separatrix. The exact interplay of the perpendicular heat source, the local particle and impurity sourcing and the parallel transport will be investigated in the future with comparative studies using EMC3EIRENE.

Indications that magnetic islands can have their own confinement was already observed in tokamaks' islands, for example at JET [20] and TEXTOR [21]. However a major difference is that those islands where located far from the particle source region (neutrals and impurities from the divertor targets), while in the island divertor the source of neutrals and particles is immediately adjacent to the island flux surface region.

In addition, the location of the local $T_{\mathrm{e}}$ maxima observed in this scenario may be also related to the position of the change in the connection length (see Figure 1(b)). In fact the region where these high temperatures are measured overlaps with the border between the short and the long connection length domain. Previous observations made with divertor Langmuir probes [22] as well as the reciprocating probe [16] correlated the highest $T_{\mathrm{e}}$ in the SOL with the position of the border between the short and the long connection length region.

For the case with $I_{\mathrm{cc}}=2 \mathrm{kA}$ in Figure 5(b) the He-beam $T_{\mathrm{e}}$ map features an overall decrease of the temperatures in the whole divertor region investigated. Moreover the $T_{\mathrm{e}}$ profiles are no longer hollow as in the previous case, but monotonically increasing moving inwards from the target. The coldest region is still around the island center, with $T_{\mathrm{e}} \sim 15 \mathrm{eV}$, which is now shifted towards the target along with the island itself. Measurements from the first LoS close to the target are missing in this scenario because of too low temperatures $\left(T_{\mathrm{e}}<10 \mathrm{eV}\right)$ to be measured by $\mathrm{He}$ emissions (which are very weak below $10 \mathrm{eV}$ ).

With application of positive current in the control coils, the island size is increased, the O-point is shifted towards the target and the island outer separatrix on the right side is pushed towards the baffle (see Figure 1(a)). The connection lengths diminish in the divertor region by $40 \%$ (see Figure $1(c)$ ). These effects can explain the global reduction of $T_{\mathrm{e}}$ in the divertor island. A drop in the connection length increases the effectiveness of the fast parallel losses and reduces the perpendicular heat transport to the island domain which globally decreases the $T_{\mathrm{e}}$ in the divertor region. Additionally, with a bigger island the parallel flow of heat and particles from the confined region streaming along the outer separatrix is shifted away from the He-beam view towards the baffle, such that local $T_{\mathrm{e}}$ maxima are no longer present in the measured profiles.

In order to qualitatively understand how the island size and connection length influence the parallel-toperpendicular transport ratio in the SOL, we consider the simple 1D SOL model developed in [23]. In this model the ratio of the parallel to perpendicular heat flux is given by $q_{\|} / q_{\perp}=\kappa_{\mathrm{e}} T_{\mathrm{e}}^{5 / 2} \Theta^{2} / \chi n_{\mathrm{e}}$, where $\chi$ is the cross-field heat conductivity assumed to be $2 \mathrm{~m}^{2} \mathrm{~s}^{-1}$ and $\kappa_{\mathrm{e}}$ the electron parallel conductivity coefficient. The field-line pitch $\Theta$ is defined as $\Theta=2 \Delta x / L_{c}$, where $\Delta x$ is the island radial width. By entering in the above expression the quantities for the two experimental scenarios, $I_{\mathrm{cc}}=0$ and $2 \mathrm{kA}$, it results that in case of $I_{\mathrm{cc}}=0 \mathrm{kA}$ the ratio $q_{\|} / q_{\perp}$ ranges between 0.3 and 2.5 moving from above the O-point to the separatrix, whereas in case of $I_{\mathrm{cc}}=2 \mathrm{kA}$ the ratio ranges from 0.2 to 4.8 . This shows that in the island divertor the cross-field transport competes with the parallel one and 

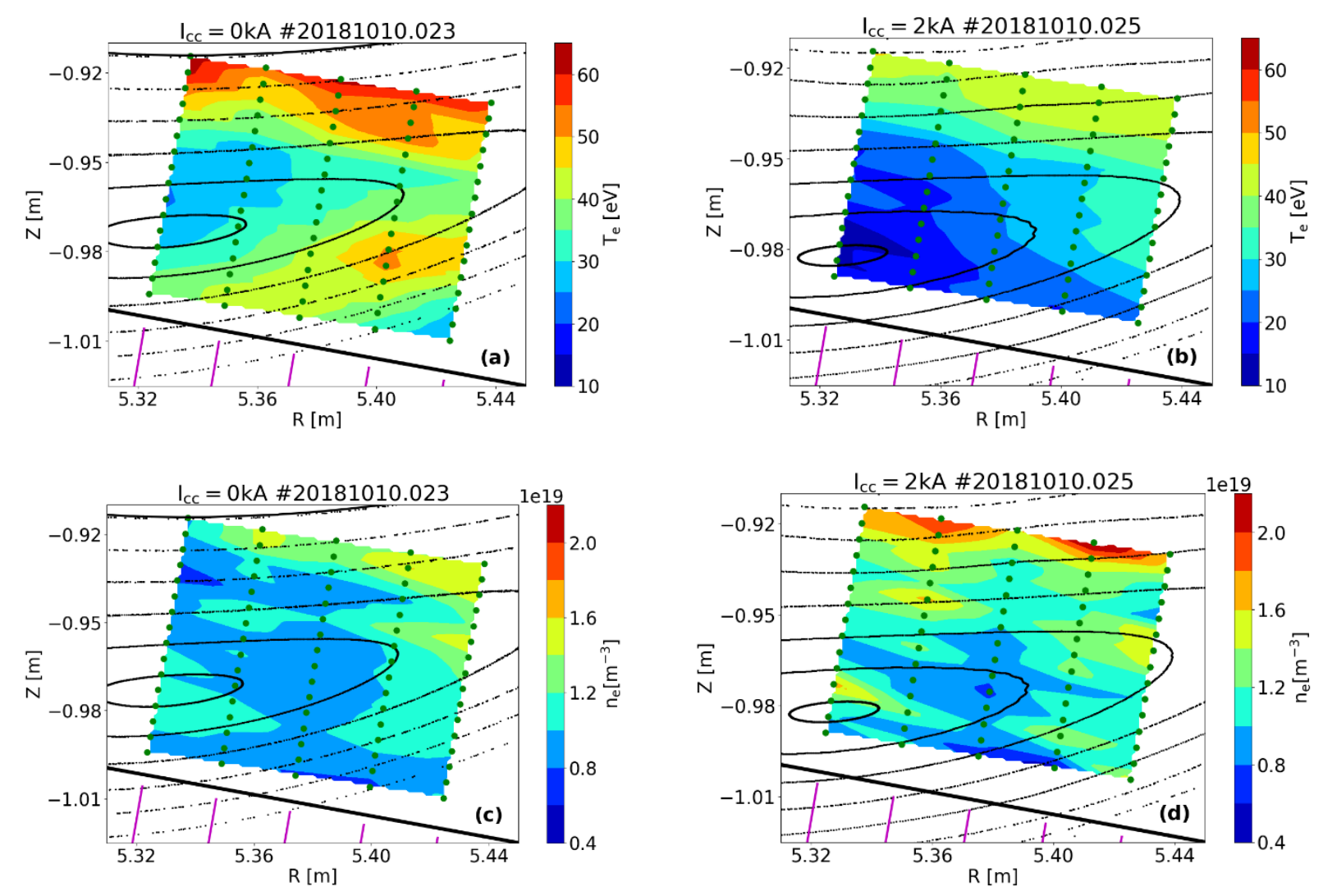

even dominates close to the O-point. With the application of the control coils and change in the connection length the weight of the parallel transport increases.

The density map measured by the He-beam is shown in Figure 5(c) and $(d)$ for both cases without and with control coils. The density distribution is found rather flat in the island region in both scenarios. We also note an increase in the density especially in the radially outward channels with application of control coils. This is likely to be a result of redistribution of the recycling particle source due to the shift in the strike line position and change of the island shape, and increased ionization length due to the overall decrease of the temperature in the divertor region. The rather flat density profiles are compatible with the results of a simple diffusive local particle balance model [24], in which the source of particles into a domain with open field lines like the island is balanced by a parallel loss to the targets. Here, particles recycle and refuel this cycle. The density profile decay length in this simple SOL model is determined by the ratio of perpendicular diffusivity and magnetic connection length to the parallel speed defining the loss rate from the region, according to the following formula: $\lambda=\sqrt{D_{\perp} L_{\mathrm{c}} / 0.5 c_{\mathrm{s}}} . D_{\perp}$ is the perpendicular diffusion coefficient assumed to be $2 \mathrm{~m}^{2} \mathrm{~s}^{-1}$ and $c_{\mathrm{s}}$ the ion sound speed equal to $5 \times 10^{4} \mathrm{~m} \mathrm{~s}^{-1}$ for $T_{\mathrm{e}}$ $\sim T_{\mathrm{i}}=20 \mathrm{eV}$. With a magnetic connection length of $300 \mathrm{~m}$ in the island domain we get a decay length greater than $10 \mathrm{~cm}$. This is compatible with the actual characteristic radial decay length in the domain covered by the He-beam. The validity of this simple SOL model in the 3D flux tube geometry of the island divertor is not immediately clear. In other 3D edge plasma studies as for instance [25], it was seen, however, that for the open field line domain comparable physics terms can be used to interpret measured plasma edge profiles across islands and stochastic magnetic field domains. 
Figure 5. 2D maps of $T_{\mathrm{e}}(R, Z)(a)(b)$ and $n_{\mathrm{e}}(R, Z)(c)(d)$ obtained by combining together the radial profiles from the He-beam at different poloidal positions for the configuration without $(a)(c)$ and with $(b)(d)$ application of control coils. Green dots represent the position of the measured points for each of the 5 valves. The five nozzles are drawn in magenta and the horizontal target in black. Poincare plots for the two configurations are shown as black dots.

\section{Temperature and density in the upstream magnetic island}

The upstream plasma profiles measured with the mid-plane reciprocating probe are depicted in Figure 6 . Here in Figure 6(a) we show the temperature profiles $T_{\mathrm{e}}(R)$ measured in two discharges with $I_{\mathrm{cc}}=0$ and $2 \mathrm{kA}$. In the scenario with $I_{\mathrm{cc}}=0$, the first important observation is that this measurement supports the observation of hollow $T_{\mathrm{e}}$ profiles when measuring across the island. In particular, a clear correlation between $T_{\mathrm{e}}(R)$ and the regions in the magnetic structure that define the edge of $\mathrm{W} 7-\mathrm{X}$ is seen. $T_{\mathrm{e}}$ is increasing in the shadowed region characterized by low $L_{\mathrm{c}}$. When approaching the domain of long $L_{\mathrm{c}}$, the temperature starts decreasing and keeps doing so while the probe plunges through the island (see Figure 2). Hollow $T_{\mathrm{e}}$ profiles measured by electric probes mounted on the reciprocating manipulator in the standard configuration were also reported in previous publications [16] [18] [26]. In the case with control coils a global temperature reduction is observed with the reciprocating probe, similarly to what was found with the He-beam. In the shadowed region $T_{\mathrm{e}}$ remains quite low, reaching $13 \mathrm{eV}$ when approaching the region of long connection length. A local maximum is still present near the border between the two magnetic domains. Then $T_{\mathrm{e}}$ slightly decreases as soon as the probe plunges into the region of long $L_{\mathrm{c}}$. The temperature rises again when moving further inwards.

The density profiles from these measurements are shown in Figure 6(b). An increasing density from the far SOL up to the island and a slight flattening is measured by the reciprocating probe when plunging through the island. This finding is not altered by a change in $I_{\mathrm{cc}}$. For the mid-plane island the flattening of the density profile inside the island may be attributed to the island topology which short circuits radially separated points leading to a flattening of the density profile. Flat density profiles inside magnetic island were observed in other stellarators like LHD [27] and HSX [28]. Also, the close proximity to the neutral and impurity source region needs to be considered. While the reciprocating probe measures upstream, under isobaric conditions, the strong sourcing downstream will distribute along the entire flux channel. Hence, the local source at the divertor target plate can overcome any parallel or perpendicular particle losses from the domain of good flux surfaces. This would yield flat density profiles as well.

In comparison with the He-beam, the temperatures measured by the reciprocating probe system are systematically lower. This will be shown explicitly in the section on Mapping the edge diagnostics together where the two diagnostics will be plotted together. This discrepancy is not understood at the moment. It isn't thought to be related to the physical mechanisms governing the divertor and the mid-plane islands, in fact from the modeling one would expect either comparable temperatures or lower downstream temperatures than upstream ones depending of the plasma conditions $\left(n_{\mathrm{es}}\right.$ and $P_{\mathrm{in}}$ ). The mismatch is rather considered to be due to an over/under estimate of $T_{\mathrm{e}}$ from one of the two diagnostics. Concerning the He-beam one of the main sources of uncertainty is the atomic model employed. Uncertainty from the atomic model can be as high as $30 \%$ [11]. Moreover, different atomic models generate substantial different temperatures [10]. From the probe side it has been observed that the measured temperature varies with the manipulator mounting different probe heads in comparable plasma conditions [16]. Concerning the density, the values measured in the divertor island with the He-beam are higher than in the midplane island measured with the reciprocating probe. The presence of the particle source at the target location may explain the higher density values measured with the He-beam with respect to the upstream probe. 


\section{Tracing the reciprocating probe to the He-beam poloidal plane}

In order to compare the upstream measurements of the reciprocating probe with the downstream ones of the Hebeam, we trace the plunging path of the reciprocating probe along the magnetic field lines to the He-beam poloidal plane, as depicted in Figure 3. Only the portion of the pathway with $R<6.08 \mathrm{~m}$ can be traced up to the He-beam location; flux tubes at larger $R$ intersect the targets before reaching the He-beam poloidal plane. In this plane we plot the $T_{\mathrm{e}}, n_{\mathrm{e}}(R, Z)$ measured by the He-beam and we superimpose as colored circles the $T_{\mathrm{e}}, n_{\mathrm{e}}(R, Z)$ from the reciprocating probe along the projected probe path: the result is shown in Figure 7. The data from both diagnostics are normalized to their respective maximum values along the projected probe's path and are plotted with the same color code, allowing for a direct comparison of the two diagnostics. For this comparison, we focus on the qualitative trend and shape of the SOL profiles rather than on the absolute numbers, which were already found to differ for the two diagnostics as discussed before. In Figure $7(a)$, the normalized distribution of $T_{\mathrm{e}}(R, Z)$ with $I_{\mathrm{cc}}=$ $0 \mathrm{kA}$ is shown. In Figure 7(b) the same is shown for the case with $I_{\mathrm{cc}}=2 \mathrm{kA}$. With this comparison it can be seen that the shapes of the profiles measured by the two diagnostics are in qualitative agreement in both scenarios. In particular the local $T_{\mathrm{e}}$ maxima observed without control coils lie along the same flux surface. The same qualitative agreement of the profile shape holds also for the plasma density map $n_{\mathrm{e}}(R, Z)$ shown in Figure $7(c)$ for the $I_{\mathrm{cc}}=0$ kA case and in Figure $7(d)$ for the $I_{\mathrm{cc}}=2 \mathrm{kA}$ case. Because the density profiles are rather flat, no accentuated

profile features can be compared, but the marginal slope across the island is found in both diagnostics.

Figure 6. Electron temperature $(a)$ and density $(b)$ profiles measured by the mid-plane reciprocating probe in the standard magnetic configuration without (blue) and with (red) application of control coils. The different domains in the magnetic structure, i.e. the SOL and the shadowed region, are labelled.
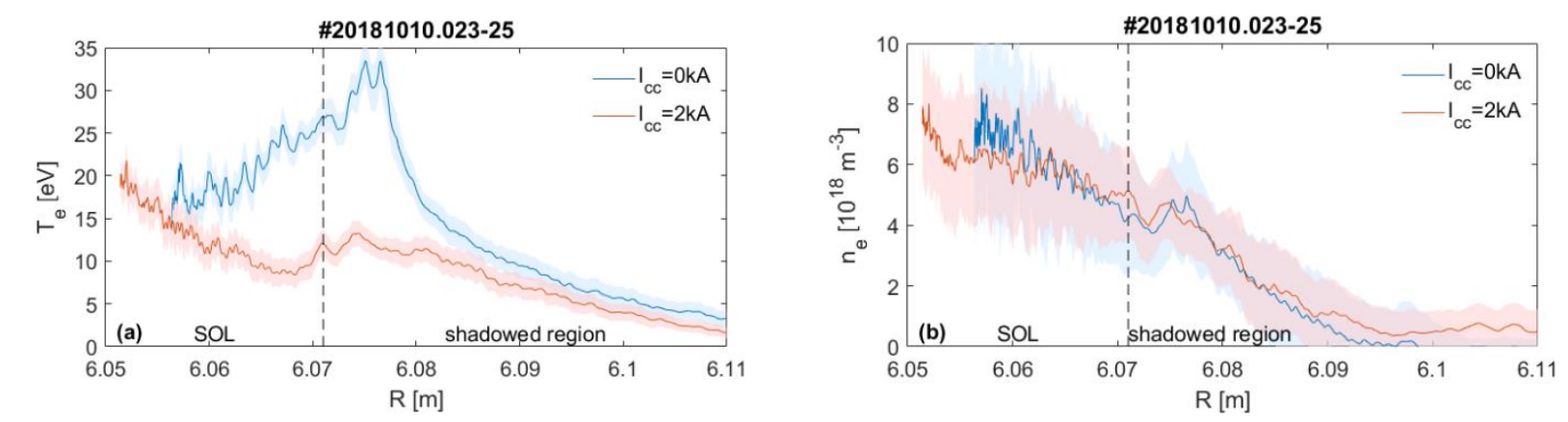

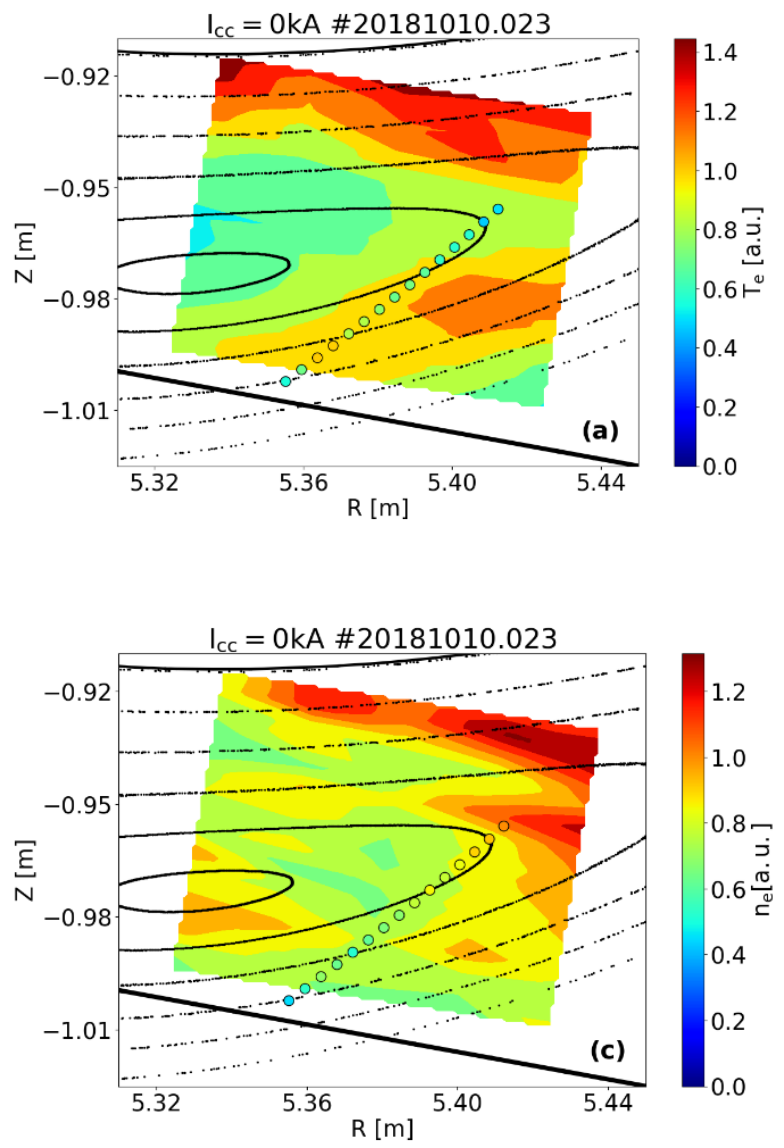
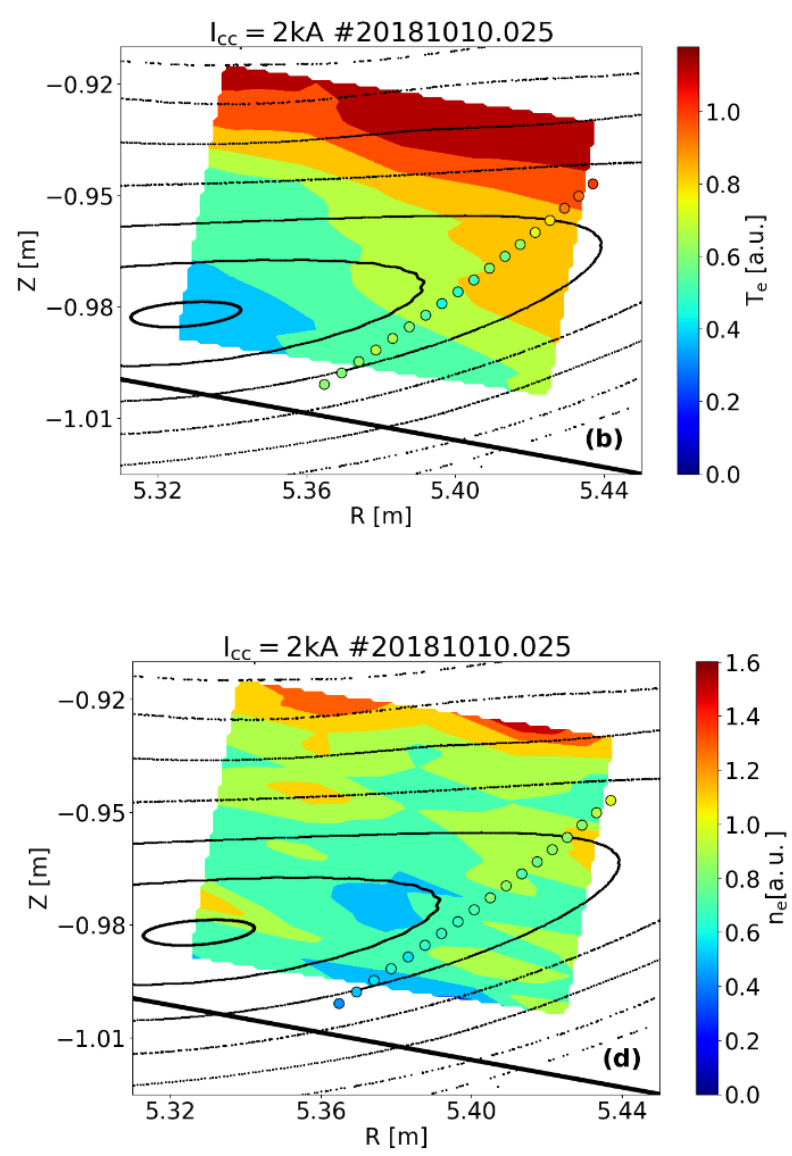

Figure 7. 2D maps of $T_{\mathrm{e}}$ and $n_{\mathrm{e}}$ measured by the He-beam with a superimposition of the $T_{\mathrm{e}}$ and $n_{\mathrm{e}}$ profiles measured by the reciprocating probe along the probe's path projected on the He-beam poloidal plane. The data from the two diagnostics are normalized to their respective maximum values along the projected probe's path. Poincare plots for the two configurations are shown as black dots.

\section{Mapping the edge diagnostics together}

In order to map edge diagnostics that are located in different flux tubes together, like for instance the He-beam and the edge channels of the Thomson scattering, we need to find a common coordinate system. This is particular challenging in the 3D magnetic structure that characterizes the SOL of W7-X. The approach that we use here is based on tracing the different diagnostics to a common poloidal plane and then using the $R_{\text {eff }}$ mapping on this plane. The chosen common poloidal plane is the one defined by the He-beam. We again trace the plunging path of the mid-plane reciprocating probe along the magnetic field lines to this plane, as depicted in Figure 3. We also trace the edge channels of the Thomson scattering system to this poloidal plane. The tracing is performed taking into account the current in the control coils as well as the toroidal plasma current. The $R_{\text {eff }}$ mapping is then performed using a VMEC magnetic equilibrium for an enlarged volume extended outside the LCFS. The islands are ignored in this computation, instead well-defined flux surfaces are assumed to exist in the island region. $R_{\text {eff }}$ is computed as $\sqrt{\langle A\rangle / \pi}$, where $\mathrm{A}$ is the cross-section of the flux surface where the point lies averaged over the torus. This approach to compute $R_{\text {eff }}$ is not considered the optimal one since VMEC magnetic equilibrium does not consider the islands and it is computed without plasma current and control coils. However at the moment no better solutions have been developed for this challenging task of finding a common coordinate system in the magnetic island domain. 
The results of the edge diagnostic comparison based on the canonical $R_{\text {eff }}$ values are shown in Figure 8 . The Hebeam $T_{\mathrm{e}}$ and $n_{\mathrm{e}}$ profiles are obtained by averaging the six profiles measured using the central valve. These six profiles were taken in a $0.5 \mathrm{~s}$ time window centered at $3.8 \mathrm{~s}$. We choose the central valve since its measured points are the ones that on the common poloidal plane share the most flux surfaces with the reciprocating probe. The Thomson scattering profiles are obtained by averaging over the same time window as for the He-beam. The probe profiles correspond to two plunges carried out at 2.7 and $5.1 \mathrm{~s}$. For the discharge with $I_{\mathrm{cc}}=2 \mathrm{kA}$ only the profiles of the second plunge are shown, since the ones of the first have high fluctuations.

In Figure $8(a)$, the $T_{\mathrm{e}}$ profiles in case of no control coils current are shown. In both the He-beam and the reciprocating probe measurements, a hollow $T_{\mathrm{e}}$ profile is visible. In particular the shape of the profiles and the position of the local maxima match. The absolute values of the temperature are in agreement between the He-beam and the Thomson scattering whereas, as pointed out earlier in the paper, the probe measures $T_{\mathrm{e}}$ about a factor of 2 lower. We notice that the $T_{\mathrm{e}}$ profile measured by the probe shifts inwards from the first to the second plunge. This may be related to the island shift and change in the connection length with increased toroidal plasma current. In fact the shift of the $T_{\mathrm{e}}$ profile $(3.5 \mathrm{~mm})$ is compatible with the island inward movement at the probe position $(2$ mm per kA of plasma current) [18].

For the case with $I_{\mathrm{cc}}=2 \mathrm{kA}$ shown in Figure $8(b)$, the temperatures lower for all diagnostics in the island region. The profile measured by the probe is still slightly hollow in correspondence of the island, while the He-beam measures an almost flat profile in the island.

The density profiles are shown in Figure $8(c)$ and $(d)$ and they are found to be higher in the divertor island measured with the He-beam - than in the mid-plane island - measured with the reciprocating probe. The Thomson scattering's edge channels measure in between two islands and their densities lie among the two other diagnostics. As explained earlier the presence of the particle source at the target location may explain the higher density values measured with the He-beam with respect to the upstream probe and the Thomson scattering. 

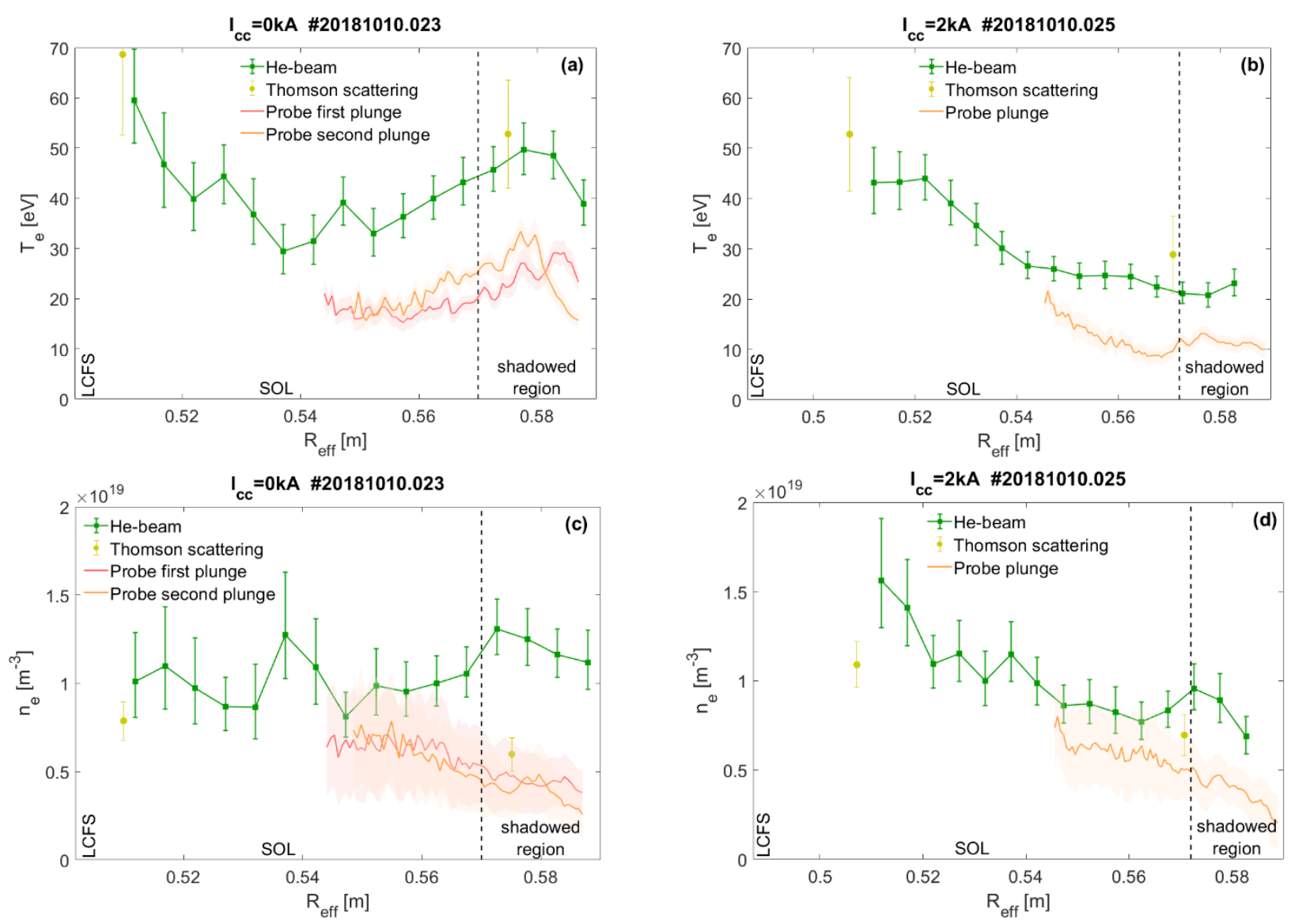

Figure 8. Mapping of the different edge diagnostics through a common radial parameter $\left(R_{\text {eff }}\right)$ as explained in the text. For the He-beam the profiles refer to the central valve. For the Thomson scattering the channels are from the low field side. $(a),(c)$ are without application of control coils, $(b),(d)$ are with. The different domains in the magnetic structure are labelled.

Discussion on the variation of the plasma parameters in the divertor island with control coils

We have already discussed two phenomena that lead to a global reduction of the temperature in the divertor island with application of control coils, such as the increase of the island size and the reduction of the connection length. Here we want to introduce the role of impurities which also contribute in reducing the temperature in this scenario. EMC3-EIRENE modeling predicts that the application of $B_{\mathrm{r}}$ produced by the control coils enhances the impurity retention effect of the island in the scenario of friction-dominated impurity transport which occurs at high density $\left(n_{\mathrm{es}} \sim 1 \times 10^{19} \mathrm{~m}^{-3}\right)$ [9]. As a consequence, increased impurity radiation contributes in cooling down the plasma. To attempt to quantitatively prove the latter effect, the impurity concentration in the divertor island would need to be inferred from divertor spectroscopy measurements. This is a challenging task because of the shape of the island and the complexity of interpreting single line emissions with respect to their functionality as an energy sink. To get a qualitative understanding of the link between impurity line radiation and the localization of the temperature reductions, one can analyze the line emission profiles from low ionization stages of carbon measured by the divertor spectroscopy. Carbon is considered to be the main impurity species in the divertor region after the application of boronization [29]. In Figure $9(a)$ and $(b)$, the C III emission profiles from the divertor spectroscopy in radial (Figure 9(a), horizontal view) and poloidal direction (Figure 9(b), vertical view) are shown. From the horizontal view we notice that the emission is concentrated near the target. This is predicted by EMC3-EIRENE modeling in a friction-force dominated scenario where the friction force exceeds the thermal force and drives the 
impurities outwards keeping them around the targets (see [3]). The peak in the emission deduced from the vertical view is located at the position of the strike line on the horizontal target. With application of $I_{\mathrm{cc}}=2 \mathrm{kA}$, the impurity radiation increases in the divertor region. This enhancement is evident at the strike line due to the shorter connection lengths which result in a faster parallel loss of heat and stronger concentration of loads on the target. More importantly, the radiation enhancement is visible also away from the strike line across the whole width of the divertor target seen by the vertical view. To relate this increased emission to a variation in the carbon concentration we would need to use for instance the S/XB formalism to transform photon fluxes into carbon fluxes. This complex analysis task is left for future research. However, it shall be noted that the measurements presented here are a self-consistent input to extract the impurity density profiles.

The bolometer diagnostic may give more insights about the localization of the SOL radiation. The bolometer at $\mathrm{W} 7-\mathrm{X}$ is installed in the triangular mid-plane, away from the divertors [30]. Its LoS cover the whole plasma crosssection including the SOL islands. The impurities are eroded from the nearby targets and then released in the islands covered by the bolometer. Preliminary tomographic reconstructions of the radiation distribution in the standard magnetic configuration show that at low radiated power (20\% of $P_{\mathrm{in}}$, as in the discharges presented here) there is evidence of localized radiation inside the islands. Further experimental efforts are needed to discern whether the radiation comes from the O-point or its surroundings.

Regarding the density variation with application of control coils, the He-beam measurements reveal a density raise in the channels away from the target with $I_{\mathrm{cc}}$. EMC3-EIRENE modeling predicts an enhancement of the downstream density with application of control coils [23] as well as an increase of recycling neutrals [9]. An according increase in the neutral pressure with $I_{\mathrm{cc}}$ was seen that supports this expectation [31]. Moreover, the temperature reduction observed near the target with application of control coils increases the ionization length of neutrals, which now travel a longer distance before getting ionized, thus increasing the density away from the target.

The findings described in this work were observed in the standard magnetic configuration in the attached divertor plasma regime, in which the downstream density is lower than the separatrix density. When the downstream density increases beyond the separatrix density we enter in the detached divertor regime characterized by high recycling neutrals. This regime cannot be characterized with the present setup of line-ratio spectroscopy on helium since the temperature in front of the target drops significantly and the He lines are no longer visible (He I lines are very weak for $T_{\mathrm{e}}<10 \mathrm{eV}$ or not detectable for $T_{\mathrm{e}}<5 \mathrm{eV}$ ). Line-ratio spectroscopy on neon is under development at W7-X in order to extend the applicability of the diagnostic to the detached divertor regime [32] [10]. 

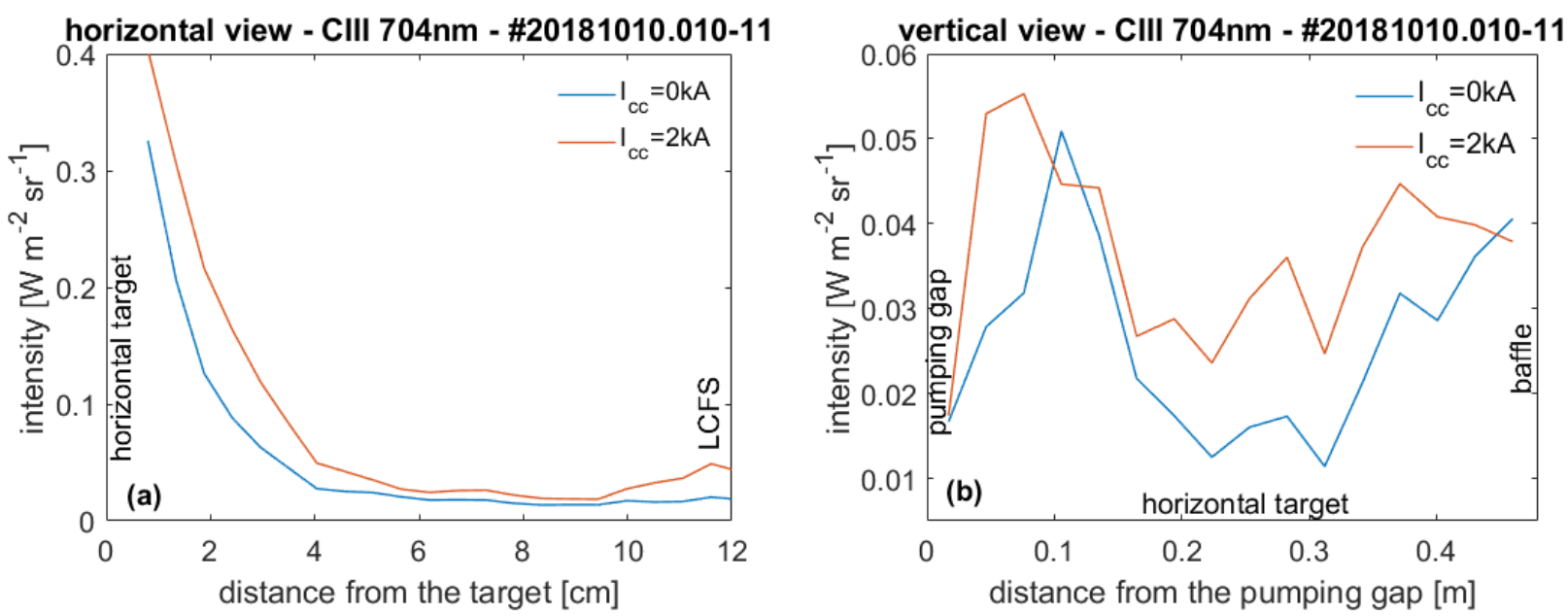

Figure 9. C III $704 \mathrm{~nm}$ line-integrated emission from the divertor region for two identical discharges with and without application of control coils. (a) horizontal view (port AEI30). LCFS lies at 9-10 cm from the target with no $I_{\mathrm{cc}}$ and at $11-12$ $\mathrm{cm}$ with $I_{\mathrm{cc}}=2 \mathrm{kA}$. (b) vertical view (port AEF30).

\section{Conclusions}

In this work, a first characterization of the plasma electron temperature and density in the divertor island is presented. The measurements were obtained with the He-beam diagnostic in the standard divertor configuration of W7-X. This diagnostic enables to measure $T_{\mathrm{e}}, n_{\mathrm{e}}$ in a broad region across the island, both in the radial and poloidal direction, creating a $2 \mathrm{D}$ map of the plasma parameters in the $R, Z$ plane. It has been shown that in the standard configuration, a zone of cold plasma is present around the O-point of the island. This region features closed flux surfaces as a sub-domain of the island, into which only perpendicular energy flux can occur. This limits the heating of this domain, which simultaneously is sourced by neutral particles and impurities from the target, situated in close proximity. The $T_{\mathrm{e}}$ profiles are hollow: when moving from the target towards the LCFS they feature a local maximum located between the island outer separatrix and its center, and a local minimum near the island center.

With application of the divertor control coils, the size of the magnetic island is increased, the connection lengths are reduced and the O-point is shifted towards the target, when compared to the case without $I_{\mathrm{cc}}$ discussed before. In this configuration the cold region in the island center enlarges and moves towards the target. Consequently the $T_{\mathrm{e}}$ profiles become monotonically increasing. Divertor spectroscopy shows that in this scenario impurity emission at the target is enhanced, in agreement with predictions from EMC3-EIRENE in a scenario of friction-dominated impurity transport.

The electron density is observed to be flat in the island width investigated by the He-beam. Density is seen to slightly increase in the external part of the island with application of the control coils, most likely due to enhanced recycling and increased ionization length.

The profile shapes measured with the He-beam in the divertor region are in good agreement with measurements carried out in an upstream location with a reciprocating probe. In particular a hollow $T_{\mathrm{e}}$ profile is also measured by the probe in case of no control coils current, and when mapping the two diagnostics together the position of the local maxima coincide. 


\section{References}

[1] R. König et al, "The divertor program in stellarators," Plasma Phys. Control. Fusion, vol. 44, p. 2365, 2002.

[2] J. Geiger et al, "Aspects of steady-state operation of the Wendelstein 7-X stellarator," Plasma Phys. Control. Fusion, vol. 55, p. 014006, 2013.

[3] Y. Feng, F. Sardei, P. Grigull, K. McCormick, J. Kisslinger and D. Reiter, "Physics of island divertors as highlighted by the example of W7-AS," Nucl. Fusion, vol. 46, p. 807, 2006.

[4] F. Sardei et al, "Island divertor studies on W7-AS," J. Nucl. Mater., vol. 241, p. 135, 1997.

[5] H. S. Bosch et al, "Technical challenges in the construction of the steady-state stellarator Wendelstein 7X," Nucl. Fusion, vol. 53, p. 126001, 2013.

[6] H. Renner, J. Boscary, H. Greuner, H. Grote, D. Hildebrandt, J. Kisslinger, R. Schneider and D. Sharma, "Divertor Concept for the WENDELSTEIN 7-X Stellarator: Theoretical Studies of the Boundary and Engineering," International Atomic Energy Agency (IAEA), IAEA-CSP--19/CD, 2003.

[7] T. S. Pedersen et al, "First divertor physics studies in Wendelstein 7-X," Nucl. Fusion, vol. 59, p. 096014, 2019. 
[8] H. Renner, J. Boscary, V. Erckmann, H. Greuner, H. Grote, J. Sapper, E. Speth, F. Wesner and M. Wanner, "The capabilities of steady state operation at the stellarator W7-X with emphasis on divertor design," Nucl. Fusion, vol. 40, p. 1083, 2000.

[9] Y. Feng, C. D. Beidler, J. Geiger, P. Helander, H. Hölbe, H. Maassberg, Y. Turkin and D. Reiter, "On the W7-X divertor performance under detached conditions," Nucl. Fusion, vol. 56, p. 126011, 2016.

[10] T. Barbui et al, "The He/Ne beam diagnostic for line-ratio spectroscopy in the island divertor of Wendelstein 7-X," JINST, vol. 14, p. C07014, 2019.

[11] O. Schmitz et al, "Status of electron temperature and density measurement with beam emission spectroscopy on thermal helium at TEXTOR," Plasma Phys. Control. Fusion, vol. 50, p. 115004, 2008.

[12] J. M. Muñoz Burgos, O. Schmitz, S. D. Loch and C. P. Ballance, "Hybrid time dependent/independent solution for the $\mathrm{He}$ I line ratio temperature and density diagnostic for a thermal helium beam with applications in the scrape-off layer-edge regions in tokamaks," Phys. Plasmas, vol. 19, p. 012501, 2012.

[13] M. Griener et al, "Qualification and implementation of line ratio spectroscopy on helium as plasma edge diagnostic at ASDEX Upgrade," Plasma Phys. Control. Fusion, vol. 60, p. 025008, 2018.

[14] T. Barbui, M. Krychowiak, R. König, O. Schmitz, J. M. Muñoz Burgos, B. Schweer and A. Terra, "Feasibility of line-ratio spectroscopy on helium and neon as edge diagnostic tool for Wendelstein 7-X," Rev. Sci. Instrum., vol. 87, p. 11E502, 2016.

[15] D. Nicolai et al, "A multi-purpose manipulator system for W7-X as user facility for plasma edge investigation," Fusion Eng. Des., vol. 123, p. 960, 2017.

[16] C. Killer, O. Grulke, P. Drews, Y. Gao, M. Jakubowski, A. Knieps, D. Nicolai, H. Niemann, A. Puig Sitjes and G. Satheeswaran, "Characterization of the W7-X Scrape-Off Layer using reciprocating probes," Nucl. Fusion, vol. 59, p. 086013, 2019.

[17] Sin-Li Chen and T. Sekiguchi, "Instantaneous Direct-Display System of Plasma Parameters by Means of Triple Probe," J. Appl. Phys., vol. 36, p. 2363, 1965.

[18] C. Killer et al, "Effect of toroidal plasma currents on the Wendelstein 7-X Scrape-Off Layer," Plasma Phys. Control. Fusion, vol. 61, p. 125014, 2019.

[19] E. Pasch, M. N. A. Beurskens, S. A. Bozhenkov, G. Fuchert, J. Knauer and R. C. Wolf, "The Thomson scattering system at Wendelstein 7-X," Rev. Sci. Instrum., vol. 87, p. 11E729, 2016.

[20] J. A. Wesson, "Snakes [JET resonant localised structures]," Plasma Phys. Control. Fusion, vol. 37, p. A337, 1995.

[21] P. C. d. Vries, G. Waidmann, A. Kraemer-Flecken and A. J. H. Donne', "Temperature profile perturbations due to magnetic islands in TEXTOR," Plasma Phys. Control. Fusion, vol. 39, p. 439, 1997. 
[22] K. C. Hammond et al, "Drift effects on W7-X divertor heat and particle fluxes," Plasma Phys. Control. Fusion, vol. 61, p. 125001, 2019.

[23] Y. Feng, M. Kobayashi, T. Lunt and D. Reiter, "Comparison between stellarator and tokamak divertor transport," Plasma Phys. Control. Fusion, vol. 53, p. 024009, 2011.

[24] P. C. Stangeby, The Plasma Boundary of Magnetic Fusion Devices, IOP Publishing, 2000.

[25] O. Schmitz et al, "Identification and analysis of transport domains in the stochastic boundary of TEXTORDED for different mode spectra," Nucl. Fusion, vol. 48, p. 024009, 2008.

[26] P. Drews et al, "Edge plasma measurements on the OP 1.2a divertor plasmas at W7-X using the combined probe," Nucl. Mater. Energy, vol. 19, p. 179, 2019.

[27] K. Ida et al, "Radial electric field and transport near the rational surface and the magnetic island in LHD," Nucl. Fusion, vol. 44, p. 290, 2004.

[28] A. R. Akerson, A. Bader, C. C. Hegna, O. Schmitz, L. A. Stephey, D. T. Anderson, F. S. B. Anderson and K. M. Likin, "Three-dimensional scrape off layer transport in the helically symmetric experiment HSX," Plasma Phys. Control. Fusion, vol. 58, p. 084002, 2016.

[29] S. Sereda et al, "Impact of boronizations on impurity sources and performance in Wendelstein 7-X," Nucl. Fusion, Accepted Manuscript 2020.

[30] D. Zhang et al, "Design criteria of the bolometer diagnostic for steady-state operation of the W7-X stellarator," Rev. Sci. Instrum., vol. 81, p. 10E134, 2010.

[31] O. Schmitz et al, "Stable heat and particle flux detachment with efficient particle exhaust in the island divertor of Wendelstein 7-X," Nucl. Fusion, under review.

[32] M. Krychowiak, M. Brix, D. Dodt, Y. Feng, R. Koenig, O. Schmitz, J. Svensson and R. Wolf, "Bayesian modelling of a thermal helium beam for measurement of electron density and temperature in the W7-X divertor plasma," Plasma Phys. Control. Fusion, vol. 53, p. 035019, 2011. 